\title{
ESTUDO DA SECAGEM DA BIOMASSA DE BANANA VERDE EM SPRAY DRYER
}

\author{
Ricardo Kenji Oi $i^{1}$ \\ Elias Basile Tambourgi ${ }^{2}$ \\ Deovaldo de Moraes Jr. ${ }^{3}$
}

Resumo: A biomassa de banana verde é um componente que pode ser aplicado em uma grande variedade de alimentos industrializados por não interferir nos atributos sensoriais de outros ingredientes e apresentar propriedades funcionais, sobretudo pela presença do amido resistente. $\mathrm{O}$ campo de aplicação pode ser ampliado, inclusive para a exportação, se produzido na forma de pó. O presente trabalho apresenta um estudo da secagem de biomassa de banana verde em uma unidade não comercial (experimental) de spray dryer com atomizador rotativo. As variáveis selecionadas no procedimento experimental foram: rotação do atomizador; temperatura da alimentação e vazão da alimentação. Estabeleceram-se como resposta a massa e o teor de água do produto. Essas variáveis tiveram dois níveis de variação, o que correspondeu à realização de oito ensaios. Dentre as variáveis utilizadas nos ensaios, a rotação do atomizador foi a mais significativa para a reposta massa e teor de água, as demais apresentaram efeitos reduzidos. Neste estudo ficou comprovada a viabilidade da secagem da biomassa de banana verde em spray dryer com atomizador rotativo.

Palavras-chave: banana verde; biomassa; alimento funcional; atomização; spray dryer.

Abstract: The green banana biomass is a component that can be industrially applied to a wide variety of foods because do not interfere in the sensory attributes of other ingredients present and has functional properties, especially the presence of resistant starch. This use can be expanded, including for export, if this product be produced in powder form. This research presents a feasibility study of drying green banana biomass in a non-commercial (experimental) of spray dryer with rotary atomizer. The variables selected in the experimental procedure were: atomizer rotation; biomass temperature and biomass flow. It was defined as response the mass and the moisture content of the product. These variables had two levels of variation, which corresponded to the completion of eight tests. Among the variables used in the tests, the atomizer rotation was the most significant to response of mass and moisture content, the others showed low effects. In this study it was proved the feasibility of drying the green banana biomass in a spray dryer with rotary atomizer.

Keywords: green banana; biomass; functional food; atomization; spray dryer.

\footnotetext{
1 Departamento de Engenharia de Sistemas Químicos da Universidade Estadual de Campinas. Doutor em Engenharia Química pela Universidade Estadual de Campinas (prof_oi@ymail.com).

2 Doutor em Engenharia Hidráulica pela Escola de Engenharia de São Carlos da Universidade de São Paulo.

3 Doutor em Engenharia Química pela Escola Politécnica da Universidade de São Paulo
} 


\section{INTRODUÇÃO}

A necessidade de melhoria da saúde leva os consumidores a buscar alimentos específicos ou componentes alimentares fisiologicamente ativos, também denominados alimentos funcionais. Para Hasler (1998), nos últimos anos, o termo funcional, aplicado aos alimentos, tem assumido diferente conotação que é a de proporcionar um benefício fisiológico adicional, além daquele de satisfazer as necessidades nutricionais básicas.

$\mathrm{O}$ alimento ou ingrediente que contenha propriedades funcionais, além de atuar em funçōes nutricionais básicas, deverá desencadear efeitos benéficos à saúde e ainda de ser seguro para consumo sem supervisão médica, conforme a ANVISA (Agência Nacional de Vigilância Sanitária).

Nos últimos anos surgiram diversas opções para essa categoria de alimentos, dentre essas a biomassa de banana verde (banana verde cozida e processada, ausente de sabor e inodora) surge com uma opção para ser utilizada em substituição aos espessantes tradicionais como trigo, soja, fécula de mandioca e amido de milho, melhorando o valor nutricional e assumindo o sabor e o aroma dos alimentos preparados com essa substância.

Diversos trabalhos foram publicados sobre as propriedades da banana verde, os quais abordaram os efeitos benéficos sobre alguns males como câncer coloretal, diarréia, índice glicêmico, resposta insulínica, dislipidemias, doenças cardiovasculares e doença celíaca (ZANDONADI, 2009; TOPPING, FUKUSHIMA e BIRD, 2003; LANGKILDE, CHAMP e ANDERSON, 2002; ENGLYST, KINGMAN e CUMMINGS, 1992; FREITAS e TAVARES, 2005).

A biomassa de banana verde ainda é produzida artesanalmente ou semi-industrialmente, em baixa escala de produção e obtida sob a forma pastosa, e mesmo que o processamento seja asséptico, persiste a preocupação quanto à questão microbiológica, assim como a perecibilidade do material.

Uma opção para a produção em escala industrial é a secagem pelo processo spray drying.

A secagem é a transferência de um líquido que se encontra em um sólido molhado para uma fase gasosa não saturada, conforme Foust et al. (1982). A operação de secagem geralmente sucede a evaporação, filtração ou cristalização, segundo Richardson, Harker e Backhurst (2002).
De acordo com Perry e Green (1997), na operação de secagem são empregados secadores industriais conforme as características da substância a ser seca e a qualidade do produto final. Os secadores são desenvolvidos a partir de algumas condiçôes de secagem como estática, dinâmica, fluidizada e diluída.

A secagem na condição diluída ocorre através da atomização, que é definida como sendo o processo de divisão do líquido em milhões de micro gotas formando um spray, sendo que $1 \mathrm{~m}^{3} \mathrm{de}$ líquido, forma aproximadamente $2 \times 10^{12}$ gotas com diâmetro aproximado de $100 \mathrm{~m}$. O secador spray dryer funciona pelo principio da secagem diluída, aceitando fluidos bombeáveis na entrada da alimentação até o final do processo e produzindo partículas secas, sem a necessidade de se empregar elevadas temperaturas, que podem degradar os nutrientes do produto a ser seco (MASTERS, 1985).

O secador do tipo spray dryer, ilustrado na Figura 1, é amplamente utilizado na secagem industrial de diversos produtos, incluindo os alimentícios. Para Foust et al. (1982), as propriedades físico-químicas dos produtos obtidos através desses equipamentos são preservadas.

A secagem da biomassa de banana verde em spray dryer permite a remoção da água e a granulação do material, facilitando o transporte e aumentando a vida de prateleira do produto. Oi et al. (2009), Oi, Tambourgi e Moraes Jr (2010) e Oi (2011) lograram êxito na secagem de biomassa de banana verde em spray dryer.

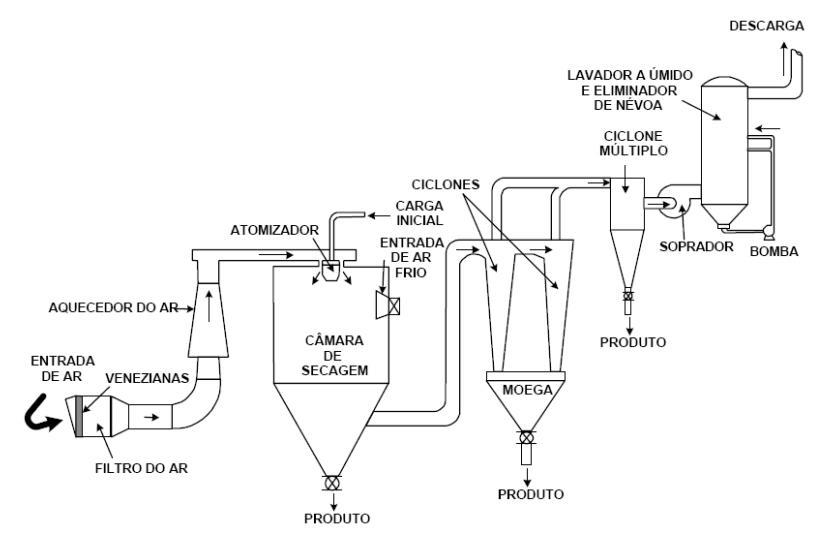

Figura 1. Instalação básica de spray dryer (FOUST et al., 1982)

$\mathrm{Na}$ secagem em spray dryer existem diversas variáveis que influenciam o processo, sendo as principais: rotação do atomizador, temperatura da 
alimentação e vazão da alimentação. Analisar essas variáveis permite para uma melhor compreensão do fenômeno, justificando assim, o desenvolvimento do presente trabalho.

Esta pesquisa teve por objetivo geral estudar a secagem da biomassa de banana verde em um spray dryer experimental com atomizador rotativo. O objetivo específico do estudo foi determinar as melhores condições operacionais nesse spray dryer, a partir da análise das principais variáveis.

\section{MATERIAIS E MÉTODOS}

\section{SPRAY DRYER}

Os ensaios para o estudo da secagem da biomassa de banana verde foram realizados em um spray dryer experimental, construído para o desenvolvimento deste trabalho, representado na Figura 2. O equipamento é constituído de uma câmara de secagem com volume de $0,2 \mathrm{~m}^{3}$, sendo o diâmetro de $0,63 \mathrm{~m}$ e a altura de $0,91 \mathrm{~m}$, fabricada em aço carbono e revestida internamente com resina polimérica (epóxi).

$\mathrm{O}$ atomizador rotativo, construído em alumínio com 30,25 mm de diâmetro e $162,88 \mathrm{~mm}^{2}$ de área dos orifícios, foi movimentado por um motor elétrico retirado de uma retífica da marca LEE de potência $127 \mathrm{~W}$, com controle de rotação e arrefecido por um cooler. Um tanque fabricado com acrílico contendo um agitador armazenou e homogeneizou a suspensão, que foi aquecida, antes de ser atomizada, em um trocador de calor constituído de tubo duplo envolto por resistência elétrica e isolante térmico. Uma bomba peristáltica composta de um rotor de alumínio com quatro roletes e acionado por um motor elétrico com controle de rotação injetou a suspensão no atomizador. Dois aquecedores, instalados na câmara em lados opostos, de resistência elétrica da marca Steinel modelo HL1800E, com potência de 2.000 W cada aqueceram o ar de secagem. O painel de controle utilizado foi da marca Salviterm modelo 704 com quatro canais.
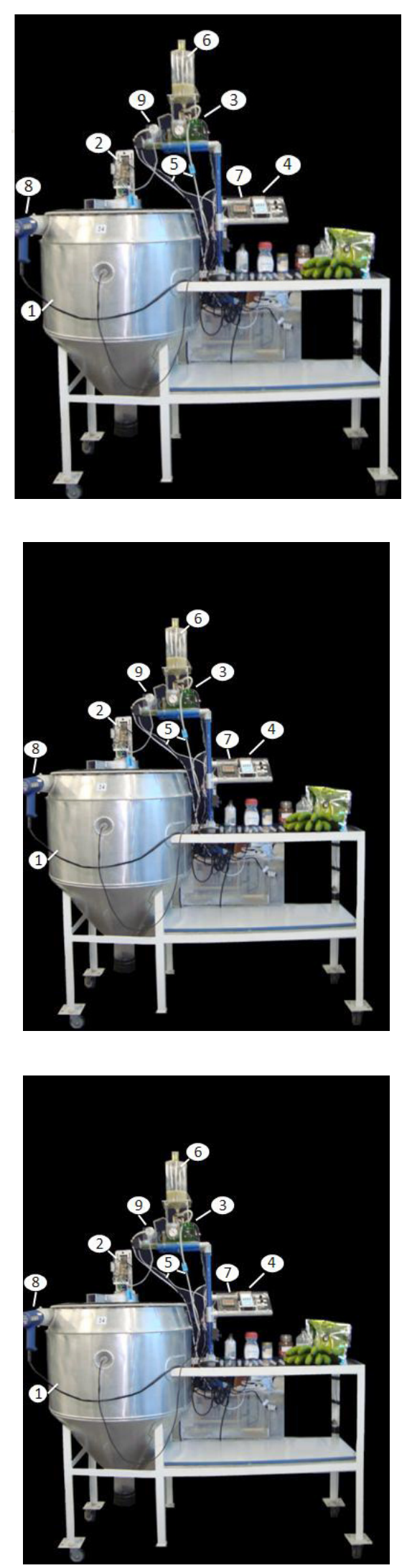

Figura 2 - Spray dryer experimental

1) Câmara de secagem; 2) Motor elétrico; 3) Bomba peristáltica; 4) Indicador de temperatura; 5) Mangueiras de silicone para o transporte da suspensão; 6) Tanque de alimentação; 7) Controlador de vazão volumétrica; 8) Aquecedor de ar; 9) Trocador de calor para pré-aquecimento. 
PREPARAÇÃO DAS AMOSTRAS

No experimento foram utilizadas três variáveis controladas, em dois níveis de variação, o que correspondeu à realização de oito ensaios, sendo efetuado um planejamento experimental. Como respostas foram definidas a massa e o teor de água. A Tabela 1 apresenta as variáveis controladas e fixadas.

$\mathrm{Na}$ biomassa de banana verde, fabricada pela Vale Mais Alimentos, cuja forma é pastosa, adicionou-se água para evitar a obstrução no atomizador e nos dutos do equipamento. Assim, nos ensaios foi utilizada uma suspensão de pasta de banana e água na concentração de 50\%. A partir da regulagem do spray dryer nas condições de cada ensaio, coletou-se o produto durante 15 minutos.

Tabela 1. Valores das variáveis utilizadas nos ensaios.

\begin{tabular}{c|c}
\hline Descrição & Valores \\
\hline \multirow{2}{*}{ Rotação do atomizador } & $23.000 \mathrm{rpm}$ \\
\cline { 2 - 2 } Temperatura de pré- & $27.000 \mathrm{rpm}$ \\
\hline aquecimento & $30^{\circ} \mathrm{C}$ \\
\hline \multirow{2}{*}{ Vazão de alimentação } & $40^{\circ} \mathrm{C}$ \\
\cline { 2 - 2 } & $40 \mathrm{~mL} / \mathrm{min}$ \\
\hline Concentração da suspensão & $60 \mathrm{~mL} / \mathrm{min}$ \\
\hline Vazão de entrada de ar & $50 \%$ \\
\hline Temperatura da câmara & $1,5 \mathrm{~kg} / \mathrm{s}$ \\
\hline
\end{tabular}

Em seguida as amostras foram pesadas em uma balança de precisão milesimal da marca Marte modelo AL 500, registrando-se os valores, e colocadas posteriormente em uma estufa da marca Olidef modelo CZ, com a temperatura regulada em $60^{\circ} \mathrm{C}$.

As amostras foram retiradas da estufa e pesadas, em intervalo de tempo de duas horas, e quando atingiram um valor constante, registrou-se a massa do produto totalmente seco. Dessa forma obteve-se o teor de água, na base úmida $\left(\mathrm{U}=\mathrm{m}_{\mathrm{a}} / \mathrm{m}_{\mathrm{t}}^{*} 100\right)$.

\section{ANÁLISE ESTATÍSTICA}

Os valores das variáveis e das respostas foram inseridos no software MINITAB 14 para a análise estatística dos efeitos das variáveis rotação do atomizador, temperatura da alimentação e va- zão da alimentação sobre as respostas massa e teor de água.

Foram elaborados no MINITAB 14, para ambas as respostas, os gráficos de Pareto e dos efeitos das variações dos valores das variáveis. Nas análises foi utilizado o nível de significância estatística $\alpha=0,10$.

\section{RESULTADOS E DISCUSSÃO}

Os resultados dos ensaios no spray dryer estão apresentados na Tabela 2, sendo: n (rotação do atomizador); $\mathbf{T}$ (temperatura de alimentação); $\mathbf{Q}$ (vazão de alimentação); $\mathbf{m}$ (massa); e $\mathbf{U}$ (teor de água).

Tabela 2. Ensaios realizados no spray dryer experimental.

\begin{tabular}{|c|c|c|c|c|c|}
\hline Ensaio & $\begin{array}{c}\mathrm{n} \\
(\mathrm{rpm})\end{array}$ & $\begin{array}{c}\mathrm{T} \\
\left({ }^{\circ} \mathrm{C}\right)\end{array}$ & $\begin{array}{c}\mathrm{Q}(\mathrm{mL} / \\
\mathrm{min})\end{array}$ & $\begin{array}{c}\mathrm{m} \\
(\mathrm{g})\end{array}$ & $\begin{array}{c}\mathrm{U} \\
(\%)\end{array}$ \\
\hline 1 & 23000 & 30 & 40 & 2,840 & 4,23 \\
\hline 2 & 23000 & 30 & 60 & 3,720 & 3,76 \\
\hline 3 & 23000 & 40 & 40 & 3,580 & 8,10 \\
\hline 4 & 23000 & 40 & 60 & 3,660 & 5,19 \\
\hline 5 & 27000 & 30 & 40 & 2,170 & 10,92 \\
\hline 6 & 27000 & 30 & 60 & 12,370 & 59,26 \\
\hline 7 & 27000 & 40 & 40 & 9,840 & 50,91 \\
\hline 8 & 27000 & 40 & 60 & 17,760 & 59,40 \\
\hline
\end{tabular}

Dos quatro ensaios realizados com a rotação de $27.000 \mathrm{rpm}$, três apresentaram as maiores quantidades de massa $17,760 \mathrm{~g}, 12,370 \mathrm{~g}$ e 9,840 g, respectivamente nos ensaios 8,7 e 6 . Nessa mesma ordem dos ensaios, verificaram-se os maiores índices de teor de água, respectivamente, $59,40 \%, 59,26 \%$ e $50,91 \%$. As maiores quantidades de massa nas amostras estão relacionadas a uma maior presença de água, justificando-se assim os maiores teores de água.

A ANVISA através da Resolução no 344 , de 13 de dezembro de 2002, impóe um limite máximo de teor de água de $13 \%$ para a farinha de trigo.

De acordo com Manica (1997), a farinha de banana verde deve conter teor de água entre 6 a 8\%. Baixos percentuais de teor de água são favoráveis a uma maior estabilidade e vida de prateleira do produto. Cabe ressaltar que 5 dos 8 ensaios, o teor de água das amostras situou-se abaixo $\operatorname{dos} 6 \%$. 


\section{INFLUÊNCIA DAS VARIÁVEIS NA MASSA}

A Figura 3 apresenta a variação da massa em relação às variáveis do experimento, onde a linha cheia horizontal presente nos três gráficos representa o valor médio da massa no experimento $(6,993 \mathrm{~g})$.

Nos ensaios que produziram maior quantidade de massa apresentaram os seguintes valores de variáveis:

a) Rotação do atomizador de $27.000 \mathrm{rpm}$;

b) Vazão de alimentação de $60 \mathrm{~mL} / \mathrm{min}$;

c) Temperatura de alimentação de $40^{\circ} \mathrm{C}$.

Gráfico das variáveis para a massa
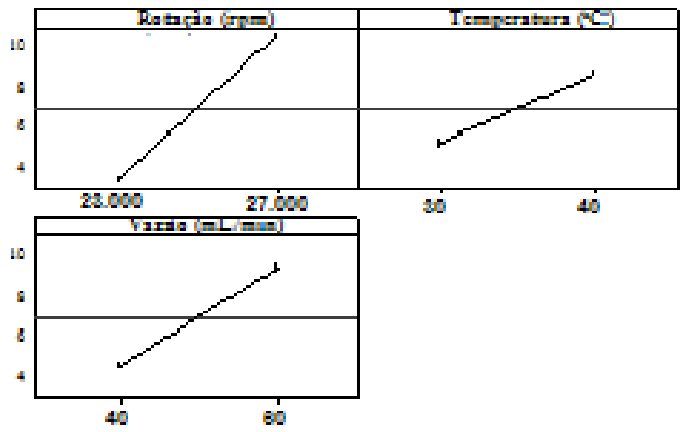

Figura 3. Efeitos das variáveis para a massa

A Figura 4 representa o gráfico de Pareto com as três variáveis para a resposta massa. A linha vertical se refere ao nível de significância estatísticapara $=0,10$.

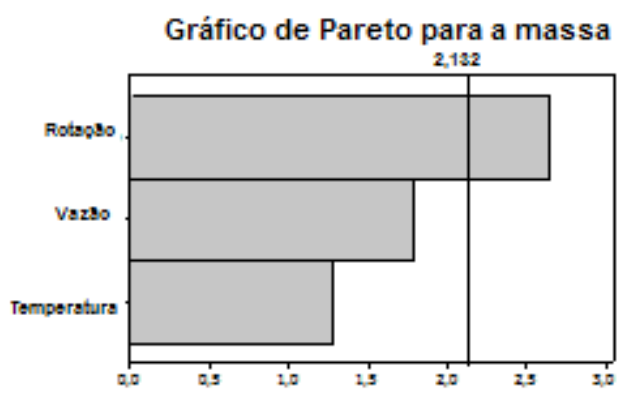

Figura 4. Gráfico de Pareto para os feitos das variáveis na massa

A rotação de 27.000 possibilitou a obtenção de amostras com maiores quantidades de massa, em comparação ao outro nível ensaiado, pois a maior velocidade da suspensão durante a atomização favoreceu uma melhor pulverização na câmara.

A vazão volumétrica de $60 \mathrm{~mL} / \mathrm{min}$, isto é, maior volume por unidade de tempo de suspensão passando pelo atomizador em relação ao outro nível testado, possibilitou a obtenção de maior quantidade de massa seca do produto.
A temperatura de $40^{\circ} \mathrm{C}$ gerou amostras com maior quantidade de massa. Uma maior temperatura de alimentação permitiu melhor eficiência na pulverização, otimizando o processo de secagem.

Das três variáveis utilizadas nos ensaios, a rotação foi aquela que mais influenciou na resposta massa, sendo a única que apresentou significância estatística no fenômeno.

\section{INFLUÊNCIA DAS VARIÁVEIS NO TEOR DE ÁGUA}

A variação do teor de água do produto em relação às variáveis do experimento está ilustrada na Figura 5. A linha cheia horizontal presente nos três gráficos representa o valor médio do teor de água $(25,22 \%)$.

\section{Gráfico das variáveis para o teor de água}
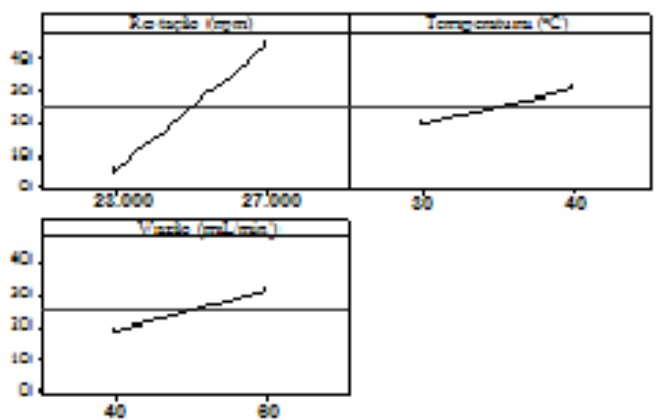

Figura 5. Efeitos das variáveis para o teor de água

Os níveis das variáveis que produziram menor teor de água foram:

a) Rotação do atomizador de $23.000 \mathrm{rpm}$;

b) Vazão de alimentação de $40 \mathrm{~mL} / \mathrm{min}$;

c) Temperatura de alimentação de $30^{\circ} \mathrm{C}$.

A Figura 6 ilustra o gráfico de Pareto com as três variáveis utilizadas no experimento para o teor de água com nível de significância estatística $=0,10$.

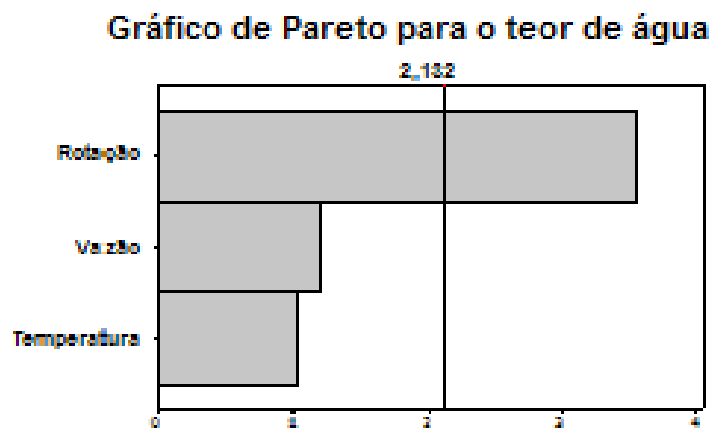

Figura 6. Gráfico de Pareto para os feitos das variáveis no teor de água 
Nos ensaios com rotação de $23.000 \mathrm{rpm}$ houve menor impregnação de material seco nas paredes da câmara, portanto, maior eficiência na secagem, e dessa forma, foi possível obter amostras com menor teor de água.

O menor volume de suspensão pulverizado na câmara, por unidade de tempo, contribuiu para uma secagem de maior eficiência, produzindo assim um produto com menor teor de água.

A temperatura de $30^{\circ} \mathrm{C}$ gerou amostras com menor teor de água. A combinação com as duas outras variáveis possibilitou que a temperatura de $40^{\circ} \mathrm{C}$ produzisse amostras com menor teor de água.

A rotação do atomizador foi a variável que mais influenciou na variação de teor de água, sendo a única que apresentou significância estatística no experimento.

Em nenhum dos ensaios houve problemas com a obstrução dos dutos de alimentação e dos orifícios do atomizador rotativo.

Nos oito ensaios realizados no experimento pôde-se comprovar a viabilidade da secagem da biomassa de banana verde em spray dryer com atomizador rotativo, corroborando com os trabalhos de Oi et al. (2009), Oi, Tambourgi e Moraes Jr (2010) e Oi (2011).

\section{CONCLUSÃO}

Conforme os resultados obtidos para secagem da biomassa de banana verde em spray dryer com atomizador rotativo pôde-se concluir que:

I. $\mathrm{O}$ ensaio $\mathrm{n}^{\circ} 2$ foi aquele que apresentou as melhores condições das respostas de massa e de teor de água, respectivamente, 3,720 g e $3,76 \%$, tendo os seguintes valores das variáveis: rotação 23.000 $\mathrm{rpm}$; temperatura de alimentação $30^{\circ} \mathrm{Ce}$ vazão de alimentação $60 \mathrm{~mL} / \mathrm{min}$;

II. Dentre as três variáveis utilizadas nos ensaios, a rotação do atomizador foi aquela que mais influenciou nas duas respostas, sendo a única que apresentou significância estatística no experimento com $=0,10$;

III. A rotação de $27.000 \mathrm{rpm}$ gerou amostras com maior quantidade de massa, já a rotação de $23.000 \mathrm{rpm}$ produziu produto com menor teor de água;

IV. Em todos os ensaios realizados foram obtidas amostras com a biomassa de ba- nana verde na forma de pó, comprovando-se a eficácia da secagem desse produto em spray dryer com atomizador rotativo.

\section{REFERÊNCIAS BIBLIOGRÁFICAS}

BRASIL. Resolução no 344, de 13 de dezembro de 2002. Regulamento Técnico para a fortificação das Farinhas de Trigo e das Farinhas de Milho com Ferro e Ácido Fólico. Diário Oficial da União, Brasília, 18 de dezembro de 2002.

ENGLYST, H. N.; KINGMAN, S. M.; CUMMINGS, J. H. Classification and measurement of nutritionally important starch fractions. European Journal of Clinical Nutrition, v. 46, p.S33-S50, 1992.

FOUST, A. S.; WENZEL, L. A.; CLUMP, C. W.; MAUS, L.; ANDERSEN, L. B. Princípios das Operaçôes Unitárias. 2a ed. Rio de Janeiro: Ed. Guanabara Dois, 1982.

FREITAS, M. C. J.; TAVARES, D. Q. Caracterização do grânulo de amido de banana (Musa AAA-Nanicão e Musa AAB Terra). Revista Ciência e Tecnologia de Alimentos, Campinas, 2005.

HASLER C. M. Functional Foods for Health Program, Department of Food Science and $\mathrm{Hu}-$ man Nutrition - University of Illinois, Urbana, Food Technology, p. 52-62, 1998.

LANGKILDE, A. M.; CHAMP, M.; ANDERSON, H. Effects of high-resistant starch banana flour (RS2) on in vitro fermentation and smallbowel excretion of energy, nutrients and sterols: an ileostomy study. American Journal Clinical Nutrition, v.75, p.104-111, 2002.

MANICA, I. Fruticultura tropical 4: banana. Porto Alegre: Continente, 1997.

MASTERS, K. Spray Drying Handbook. 4th. ed. London: George Godwin, 1985.

OI. R. K. Secagem da biomassa de banana verde em spray dryer. Tese (Doutorado em Engenharia Química), Faculdade de Engenharia Química, Universidade Estadual de Campinas - UNICAMP), 2011. 
OI, R. K.; TAMBOURGI, E. B.; MORAES JR, D. Estudo de viabilidade da secagem da biomassa da banana verde em spray dryer rotativo. Exacta, São Paulo, v. 8, n.2, p. 185-191, 2010.

OI, R. K.; MORAES, M. S.; FERNANDES, F. M.; MOINO, C. A. A.; TAMBOURGI, E. B.; MORAES JR, D. Projeto de secador do tipo spray dryer para secagem de biomassa de banana verde. In: $I X$ Congreso Iberoamericano de Ingeniería Mecânica, 2009, Las Palmas de Gran Canária: Airexpress.

PERRY, R. H.; GREEN, D. W. Perry's Chemical Engineers' Handbook. 7th. ed. New York: McGraw-Hill, 1997.
RICHARDSON, J. F.; HARKER, J. H. Coulson and Richardson's Chemical Engineering. Vol. 2 Technology and Separation Processes. Oxford: Butterworth-Heinemann, 2002.

TOPPING, D. L.; FUKUSHIMA, M.; BIRD, A. R. Resistant starch as a prebiotic and synbiotic: state of the art. Proceedings of the Nutrition Society, v. 62, p. 171-176, 2003.

ZANDONADI, R. R. Massa de banana verde: uma alternativa para exclusão do glúten. 2009. $74 \mathrm{f}$. Tese (Doutorado em Ciências da Saúde), Faculdade de Ciências da Saúde, Universidade de Brasília - UnB, Brasília. 